\title{
BreastCore
}

\section{Are We Ready to Use ESR1 Mutations in Clinical Practice?}

\author{
Rinath Jeselsohn ${ }^{a, b}$ \\ a Breast Oncology Center, Dana Farber Cancer Institute, Harvard Medical School, Boston, MA, USA; \\ ${ }^{b}$ Center for Functional Cancer Epigenetics, Dana Farber Cancer Institute, Harvard Medical School, Boston, MA, USA
}

\section{Keywords \\ Estrogen receptor . ESR1 mutations . \\ Endocrine resistance $\cdot$ Circulating tumor DNA}

\section{Summary}

The recurrent ligand-binding domain ESR 1 mutations are an important mechanism of endocrine resistance in estrogen receptor-positive $(E R+)$ metastatic breast cancer. These mutations evolve under the selective pressure of endocrine treatments and are rarely found in treatment-naïve ER+ breast cancers. Preclinical studies showed that these mutations lead to ligand-independent activity facilitating resistance to aromatase inhibitors and relative resistance to tamoxifen and fulvestrant. Retrospective analyses of ESR 1 mutations in baseline plasma circulating tumor DNA from clinical trials suggest that these mutations are prognostic of poor overall survival and predictive of resistance to aromatase inhibitors in metastatic disease. Larger datasets and prospective studies to confirm these results are lacking. In addition, response to other standard treatments for metastatic breast cancer in the presence of the ESR1 mutations is unknown, and studies to determine the optimal treatment combinations for patients with ESR1 mutations are also needed.

(C) 2017 S. Karger GmbH, Freiburg

\section{Introduction}

About $70 \%$ of all breast cancers are estrogen receptor(ER)-positive $(\mathrm{ER}+)$. ER is a ligand-dependent transcription factor and a member of the nuclear receptor superfamily. The protein consists of multi-functional domains including the DNA binding and hinge domains flanked by the transcriptional transactivation domains, the ligand-independent activation function-1 (AF-1), and the ligand-dependent activation function-2 (AF-2). The AF-2 overlaps with the ligand binding domain (LBD). ER undergoes conformational changes after ligand binding. These include ER dimerization and phosphorylation followed by recruitment to numerous DNA sites to regulate the transcription of genes that have key roles in proliferation and anti-apoptosis. The genome-wide distribution of ER binding sites within a particular cell, defined as the ER cistrome, and its transcriptional program are dictated by multiple factors including receptor tyrosine kinase signaling pathways, ER coregulators (co-activators and co-repressors), pioneer factors, and chromatin accessibility [1-3].

ER is a key therapeutic target in ER+ breast cancer. The most commonly used endocrine therapies inhibit ER activity by either targeting the ER protein itself or by depriving the receptor of its ligand. The different classes of endocrine treatments include: i) selective ER modulators (SERMs) which compete with the estrogen ligand and have mixed agonistic/antagonistic capacities; ii) selective ER degraders (SERDs) which possess almost exclusive antagonistic activity and induce ER protein degradation; and iii) depletion of systemic estrogen levels by aromatase inhibitors (AIs). While endocrine therapies have proven to be very effective in both the early and the metastatic setting, both de novo and acquired resistance to endocrine treatments remain a key clinical challenge [4].

\section{ESR1 LBD Missense Mutations as a Mechanism of Endocrine Resistance}

Mutations of the ESR1 gene that encodes ER were first studied in cell line models [5]. Thereafter, an ESR1 Y537N activating missense mutation was described in a single metastatic sample in 1997 [6]. However, other studies, mainly in treatment-naïve primary tu-

\section{KARGER \\ Fax +497614520714

() 2017 S. Karger GmbH, Freiburg 
mors, failed to detect the Y537N mutation or similar ESR1 mutations $[7,8]$. This includes the TCGA (The Cancer Genome Atlas) study, in which 962 primary breast cancers were sequenced, and ESR1 mutations were found in $0.5 \%$ of the samples [9]. It was only in 2013 that a series of studies using next generation sequencing reported the detection of ESR1 recurrent LBD mutations in metastatic ER+ breast cancer tissue specimens. The first study employed whole exome sequencing of patient-derived xenograft (PDX) models derived from ER+ metastatic samples. ESR1 mutations were found in 3 of the 7 models and were confirmed in the originating metastatic tumors [10-12]. A second study included 80 patients with ER+ metastatic disease, who had progressed on endocrine treatment [11]. This study found 14 patients with ESR1 LBD mutations (17.5\%). In 2 small studies, in which 11 and 13 metastatic $\mathrm{ER}+$ tumor samples were sequenced, ESR1 LBD recurrent mutations were found in 6 and 5 tumors, respectively $[12,13]$. In another study, 76 metastatic ER+ metastatic tumors were sequenced, and 11 ESR1 LBD mutations were found (14.4\%) [14]. In a more recent study, 929 breast cancer tumor samples were sequenced. The majority of these samples were ER+ metastatic tumors, with 95 patients found to have a somatic ESR1 mutation. Not surprisingly, 85 of the 95 patients had ER+ metastatic disease [15].

The recurrent ESR1 mutations were all found within the LBD. The most common mutations were the missense mutations Y537 and D538. These mutations are gain-of-function mutations that lead to constitutive, ligand-independent activation of ER [11, 13, 14]. Y537 and D538 are hotspot mutations in helix 12 (H12), a key structural part of AF-2. In wild-type (WT) ER, estrogen binding to the LBD leads to stabilization of H12 in an active conformation leading to increased binding of co-activators with subsequent activation of the receptor. Conversely, antagonist binding to the LBD induces an inactive conformation of $\mathrm{H} 12$ that promotes co-repressor binding and inhibition of the receptor activity. The Y537S and D538G ER mutations stabilize H12 in the agonistic conformation, similar to WT-ER bound to E2 [16, 17]. In addition, affinity studies showed that the LBD-mutated receptors have a decreased affinity for estradiol (E2). Finally, several studies showed co-activator binding to mutant ER in ligand-independent conditions $[15,16]$. Together, these observations provide a mechanistic explanation for the ligand-independent activity. In addition, the ligand-independent activity of the ESR1 LBD mutations, and the fact that they are more prevalent in metastatic disease after treatment with endocrine treatments, indicate that the selection of these mutations occurs under the pressure of endocrine treatment, particularly AIs.

\section{ESR1 Mutations as a Prognostic Factor in Metastatic Disease}

A number of groups have investigated the use of circulating tumor DNA (ctDNA) to detect ESR1 LBD mutations in the setting of metastatic disease in a non-invasive manner. This could eventually facilitate our understanding of the clinical significance of these mutations and the development of drugs to overcome resistance engendered by them. Thus far, there have been a number of reports of post hoc analyses of ctDNA ESR1 mutations in plasma samples obtained from patients that participated in published clinical trials of metastatic ER+ breast cancer. These studies employed droplet digital polymerase chain reaction (ddPCR) for the detection of the mutations in ctDNA, and the prevalence of the ESR1 mutations ranged between 25.3 and $39.1 \%$ [18-20]. These numbers are overall higher compared to the rates seen in the studies of metastatic tissue samples, and this likely due to the ability of plasma samples to capture the heterogeneous genomic landscape of multiple metastatic sites and the high sensitivity of the ddPCR assay. In addition, a number of the ESR $1 \mathrm{ctDNA}$ studies have now demonstrated that the presence of ESR1 mutations in metastatic disease is a poor prognostic factor $[17,18]$.

In 1 study, baseline ctDNA for the Y537S and D538G ESR $1 \mathrm{mu}-$ tations was tested in archival plasma samples collected from the participants of the BOLERO-2 study [18]. This was a phase III study in which patients with metastatic ER+ breast cancer and prior exposure to a non-steroidal AI were randomized to exemestane versus exemestane plus the mTOR inhibitor everolimus. In this study, ESR1 mutations (Y537S, D538G, or both) were detected in $28.8 \%$ of patients $(156 / 541)$. Patients with an ESR1 mutation had worse overall survival (OS) compared to WT ESR1. A subgroup analysis showed that patients with the D538G mutation alone, the Y537S mutation alone, or both mutations, all had decreased OS compared to WT ESR1. Median OS for patients with WT ESR1, the D538G mutation, the Y537S mutation, and both mutations was 32, 26, 20, and 15 months, respectively. The effect of the ESR1 mutations on OS remained significant after adjustment for confounding factors such as previous endocrine treatment, visceral disease, and performance status. In a second study, a retrospective analysis of plasma samples from patients with metastatic $\mathrm{ER}+$ disease that had developed disease progression on AI treatment was performed [21]. Among the 141 patients in this study, $30.6 \%$ were found to have an ESR1 mutation, and these patients had significantly decreased OS compared to patients with WT ESR1 (median OS 15.5 months compared to 23.8 months). Furthermore, the prognostic value of ctDNA ESR1 mutations was confirmed in a multivariate analysis in this study.

\section{ESR1 Mutations and Endocrine Therapy}

In addition to the constitutive activity of the LBD ER mutations, these mutations lead to a growth advantage in hormone-deprived conditions and relative resistance to tamoxifen and fulvestrant. This was shown in vitro as well as in vivo using breast cancer cell lines engineered to express the mutations and PDXs derived from human metastatic breast cancer tumors harboring the LBD mutations $[10,11,14]$. The crystal structure and simulation studies of tamoxifen bound to the D538G and Y537S mutants indicate that the ER LBD mutations lead to decreased affinity to tamoxifen and confer an altered antagonistic conformation facilitating resistance to antagonism by tamoxifen [17]. Fulvestrant also binds to the 
LBD; however, the structure of the mutant LBD bound to fulvestrant has not been resolved. Thus, the biochemical mechanism of the fulvestrant relative resistance observed in preclinical models has not been elucidated.

The sequencing studies of metastatic tissue samples demonstrated the association between endocrine treatments, particularly AIs, and the emergence of the LBD ESR1 mutations. More recently, a number of ctDNA studies focused on the detection of the ESR1 mutations have shed some light on the predictive value of the ESR1 mutations for subsequent endocrine treatments. In one of the first studies of ctDNA ESR1 mutations, plasma was tested for ESR1 LBD mutations (L536R, Y537C, Y537N, D538G) using ddPCR in 128 patients with advanced $\mathrm{ER}+$ breast cancer at the time of disease progression [22]. ESR1 LBD mutations were detected in $14.8 \%$ of these patients and were found exclusively in patients who had prior treatment with an AI. An analysis of a limited number of patients with and without detectable ctDNA ESR1 mutations showed that the ESR1 mutations are associated with a shorter progression-free survival (PFS) on subsequent AI treatment. In a second study, plasma samples from 69 patients with metastatic ER+ disease were retrospectively analyzed for Y537S/N and D538G mutations. Patients with an ESR1 mutation had decreased PFS during subsequent endocrine treatment compared to patients with WT ESR1. In this analysis, a number of endocrine treatments were grouped together, including AIs, SERMs, SERDs, ethinyl estradiol, and medroxyprogesterone acetate [23]. Thus, it is difficult to interpret the predictive value of the ESR1 mutations for a specific endocrine treatment from the results of this study. A number of groups performed post hoc prospective-retrospective analyses of ESR1 mutations in plasma samples from completed randomized clinical trials examining the impact of the ESR1 mutations on response to subsequent treatment with AIs and fulvestrant [18-20]. In the first study, baseline plasma samples from the FERGI study were analyzed [20]. The FERGI study was a randomized phase II study that compared pictilisib, a pan-PI3K kinase inhibitor, combined with fulvestrant compared to fulvestrant plus placebo in patients with metastatic ER+ breast cancer who had progressed on AI treatment. Baseline ctDNA was analyzed for 12 ESR1 (E380Q, S463P, V534E, P535H, L536P, L536H, L536H, Y536S, Y536N, Y537C, D538G) and 9 PIK3CA mutations. ESR1 mutations were detected in $37 \%$ of plasma samples (78/207). In this study, the presence of the ESR1 mutations did not impact the clinical benefit from fulvestrant treatment. In the second study, baseline plasma samples from a subgroup of participants in the SoFEA phase III clinical trial were tested for ctDNA ESR1 mutations (E380Q, L536R, Y537C, D538G, S463P, Y537N, Y537S) [19]. The SoFEA study included patients with metastatic ER+ disease that had prior exposure to a non-steroidal AI. Patients were randomized to fulvestrant plus anastrozole, fulvestrant plus placebo, or exemestane. ESR1 mutations were detected in $39.1 \%$ of the patients in this trial (63/161). Patients with an ESR1 mutation had improved PFS with a fulvestrant-containing treatment compared to exemestane alone (median PFS 2.6 months for exemestane and 5.7 months for fulvestrant; hazard ratio (HR) $0.52, \mathrm{p}=0.02)$. In contrast, for patients with WT ESR1, there was no significant difference in PFS between exemestane and fulvestrant treatment (median PFS 8 months for exemestane and 5.4 months for fulvestrant; HR 1.07, p = 0.77). However, the interaction test between treatment arm and ESR1 mutation status was not statistically significant. Overall, these results suggest that patients with an ESR1 mutation might benefit more from a fulvestrant-containing regimen rather than a regimen with exemestane. However, confirmatory studies with a larger number of patients with ESR1 mutations are still needed.

\section{Emerging Endocrine Treatments}

Since the ESR1 mutations are activating mutations, they are likely important treatment targets. Preclinical studies showed that mutant ER has a decreased affinity to tamoxifen, and higher concentrations of tamoxifen and fulvestrant can inhibit the ER mutations. These results imply that higher doses of either tamoxifen or fulvestrant could potentially inhibit the ER mutations. Alternatively, newer SERMs/SERDs with improved pharmacologic properties compared to fulvestrant may have improved efficacy in inhibiting the ER mutations.

Bazedoxifene is a third-generation SERM with SERD activity [24], and in a preclinical study of a Y537S ER mutation PDX model, bazedoxifene as a single agent or in combination with palbociclib effectively reduced tumor growth and Ki67 levels [25]. In addition, biophysical and structural studies showed that bazedoxifene increases the dynamics of $\mathrm{H} 12$ resulting in improved potency against the ER mutations [26]. Elacestrant (RAD1901) is an orally bioavailable small-molecule SERD. Preclinical studies showed inhibition of tumor growth in PDX models harboring the ER mutations with elacestrant [27]. Recently reported was the analysis of 40 patients with metastatic ER+ breast cancer that received a 400-mg dose of elacestrant in a phase I dose escalation study followed by a safety expansion cohort. Inclusion criteria included progression after more than 6 months on prior endocrine treatment. Of these 40 patients, $50 \%$ were found to have an ESR 1 mutation in cell-free DNA. For 22 patients, RECIST measurable disease was available, and among these, 5 patients with either WT ER or mutant ER had a partial response [28]. GDC-0810 is another new orally bioavailable SERD [29]. Preclinical studies in cell lines and PDX models showed that GDC-0810 inhibits tumor growth in the presence of the ESR1 mutations. In addition, a phase I dose escalation study including 41 patients with metastatic ER+ disease was reported. This study included fluoroestradiol(FES)-positron emission tomography imaging to evaluate blockage of ER, and complete or near complete suppression of FES uptake was observed in $90 \%$ of patients, including 5 patients with known ESR1 mutations [30]. A third orally bioavailable SERD, GDC0927, is currently in clinical development. A phase I study for patients with metastatic ER+ breast cancer is currently ongoing (NCT02316509). The continued development of these new and other novel endocrine treatments will likely provide more data on the efficacy of these drugs in breast cancers that harbor the ESR1 mutations. 


\section{CDK4/6 Inhibitors and ESR1 Mutations}

Over the past few years, the CDK4/6 inhibitors have emerged as an important addition to our armamentarium in the treatment of ER+ breast cancers. Thus far, 2 CDK4/6 inhibitors, palbociclib and ribociclib, have been approved for the treatment of metastatic ER+ breast cancer. Cyclin D1 in complex with CDK/4/6 is a key cell cycle regulator and is amplified and/or overexpressed in about $50 \%$ of breast cancers. Additionally, cyclin D1 is an ER transcriptional target, and there is a strong correlation between ER expression and cyclin D1 [31]. In keeping with these findings, when multiple breast cancer cell lines were tested for palbociclib sensitivity, the ER+ breast cancer cell lines had low IC50 values, and synergy with tamoxifen was observed [32]. Since cyclin D1 is an ER transcriptional target, the presence of an ESR1 mutation likely does not decrease response to CDK4/6 inhibitors. Indeed, this was seen in the PALOMA-3 study. The PALOMA-3 study was a phase III study in which patients were randomized to fulvestrant and palbociclib versus fulvestrant and placebo [3]. Baseline plasma samples from a subgroup of participants $(n=360)$ were retrospectively tested for mutations using ddPCR. ESR1 mutations were found in $25.3 \%$ of the patients (91/360). Of the patients that had an ESR1 mutation, 63 received the combination treatment (median PFS 9.4 months) and 28 received fulvestrant and placebo (median PFS 3.6 months). The patients that had an ESR1 mutation had a significantly improved PFS (HR 0.43, p = 0.002). Thus, based on this analysis, patients harboring an ESR1 mutation benefit from the addition of palbociclib.

\section{Conclusion}

The LBD activating ESR1 mutations are present in a high percentage of metastatic ER+ breast cancers. These mutations are an important mechanism of endocrine resistance and also a marker of an active ER transcriptional program. In the initial next generation sequencing studies of tissue specimens, the mutations were found in $15-20 \%$ of metastatic ER+ tumors, with a relatively higher prevalence in patients who have received multiple lines of endocrine treatment. Analysis of ctDNA, which captures the intra-tumoral heterogeneity and the heterogeneity between different metastatic sites, showed that the ESR 1 mutations are found in close to $40 \%$ of patients with metastatic ER+ breast cancer. Testing ctDNA for ESR1 mutations in the metastatic setting is a promising tool for the continued investigation of the clinical role of these mutations as a predictive biomarker. However, for clinical utility, this test will need to be standardized. Particularly, a standardized cutoff has not been established, and it is not known what mutational allele frequency is clinically significant. In addition, the majority of the studies used dichotomized results of baseline ctDNA analysis, and it is not known if the allele frequency and kinetics of the allele frequency based on serial testing are of clinical importance.

A post hoc analysis of a randomized clinical trial of patients with metastatic breast cancer after progression on first-line treatment with a non-steroidal AI showed that patients with an ESR1 mutation have worse PFS on a steroidal AI compared to fulvestrant treatment. These results provide clinical evidence for AI resistance in the presence of ER mutations and suggest that the dose of fulvestrant in this clinical trial may be sufficient to overcome the relative resistance predicted by the preclinical studies. In addition, another post hoc analysis suggested that patients with metastatic breast cancer harboring the ESR1 mutations benefit from the addition of palbociclib to fulvestrant. However, these retrospective analyses are exploratory only and are not practice changing. Prospective clinical trials in which patients are stratified on the basis of the ESR1 mutant status are needed, both to confirm these findings and to investigate the sensitivity of the mutations to other drugs such as tamoxifen, everolimus, and ribociclib. In addition, clinical trials dedicated to patients with ESR1 mutations are needed to study new SERMs/SERDs and other novel agents currently under investigation in clinical studies.

\section{Acknowledgement}

This paper was supported by the NCI Grant K08 CA191058-02 (R.J.).

\section{Disclosure Statement}

The author has no conflicts of interest to declare.

\section{References}

1 Carroll JS, Meyer CA, Song J, et al.: Genome-wide analysis of estrogen receptor binding sites. Nat Genet 2006;38:1289-1297.

2 Lupien M, Meyer CA, Bailey ST, et al.: Growth factor stimulation induces a distinct ER(alpha) cistrome underlying breast cancer endocrine resistance. Genes Dev 2010;24:2219-2227.

3 Cristofanilli M, Turner NC, Bondarenko I, et al.: Fulvestrant plus palbociclib versus fulvestrant plus placebo for treatment of hormone-receptor-positive, HER2-negative metastatic breast cancer that progressed on previous endocrine therapy (PALOMA-3): final analysis of the multicentre, double-blind, phase 3 randomised controlled trial. Lancet Oncol 2016;17:425-439.
4 Williams N, Harris LN: The renaissance of endocrine therapy in breast cancer. Curr Opin Obstet Gynecol 2014;26:41-47.

5 Weis KE, Ekena K, Thomas JA, et al.: Constitutively active human estrogen receptors containing amino acid substitutions for tyrosine 537 in the receptor protein. Mol Endocrinol 1996;10:1388-1398.

6 Zhang QX, Borg A, Wolf DM, et al.: An estrogen receptor mutant with strong hormone-independent activity from a metastatic breast cancer. Cancer Res 1997; 57:1244-1249.

7 Karnik PS, Kulkarni S, Liu XP, et al.: Estrogen receptor mutations in tamoxifen-resistant breast cancer. Cancer Res 1994;54:349-353.
8 Roodi N, Bailey LR, Kao WY, et al.: Estrogen receptor gene analysis in estrogen receptor-positive and receptor-negative primary breast cancer. J Natl Cancer Inst 1995;87:446-451.

9 Cancer Genome Atlas Network: Comprehensive molecular portraits of human breast tumours. Nature 2012;490:61-70.

10 Li S, Shen D, Shao J, et al.: Endocrine-therapy-resistant ESR1 variants revealed by genomic characterization of breast-cancer-derived xenografts. Cell Rep 2013;4: 1116-1130.

11 Toy W, Shen $\mathrm{Y}$, Won H, et al.: ESR1 ligand-binding domain mutations in hormone-resistant breast cancer. Nat Genet 2013;45:1439-1445. 
12 Robinson DR, Wu YM, Vats P, et al.: Activating ESR1 mutations in hormone-resistant metastatic breast cancer. Nat Genet 2013;45:1446-1451.

13 Merenbakh-Lamin K, Ben-Baruch N, Yeheskel A, et al.: D538G mutation in estrogen receptor-alpha: a novel mechanism for acquired endocrine resistance in breast cancer. Cancer Res 2013;73:6856-6864.

14 Jeselsohn R, Yelensky R, Buchwalter G, et al.: Emergence of constitutively active estrogen receptor-alpha mutations in pretreated advanced estrogen receptor-positive breast cancer. Clin Cancer Res 2014;20:1757-1767.

15 Toy W, Weir H, Razavi P, et al.: Activating ESR1 mutations differentially affect the efficacy of ER antagonists. Cancer Discov 2017;7:277-287.

16 Nettles KW, Bruning JB, Gil G, et al.: NFkappaB selectivity of estrogen receptor ligands revealed by comparative crystallographic analyses. Nat Chem Biol 2008; 4 : 241-247.

17 Fanning SW, Mayne CG, Dharmarajan V, et al.: Estrogen receptor alpha somatic mutations Y537S and D538G confer breast cancer endocrine resistance by stabilizing the activating function-2 binding conformation. Elife 2016;5:e12792.

18 Chandarlapaty S, Chen D, He W, et al.: Prevalence of ESR1 mutations in cell-free DNA and outcomes in metastatic breast cancer: a secondary analysis of the BOLERO-2 clinical trial. JAMA Oncol 2016;2:13101315.

19 Fribbens C, O'Leary B, Kilburn L, et al.: Plasma ESR1 mutations and the treatment of estrogen receptor-positive advanced breast cancer. J Clin Oncol 2016;34: 2961-2968.
20 Spoerke JM, Gendreau S, Walter K, et al.: Heterogeneity and clinical significance of ESR1 mutations in ERpositive metastatic breast cancer patients receiving fulvestrant. Nat Commun 2016;7:11579.

21 Clatot F, Perdrix A, Augusto L, et al.: Kinetics, prognostic and predictive values of ESR1 circulating mutations in metastatic breast cancer patients progressing on aromatase inhibitor. Oncotarget 2016;7:74448-74459.

22 Schiavon G, Hrebien S, Garcia-Murillas I, et al.: Analysis of ESR1 mutation in circulating tumor DNA demonstrates evolution during therapy for metastatic breast cancer. Sci Transl Med 2015;7:313ra182.

23 Takeshita T, Yamamoto Y, Yamamoto-Ibusuki M, et al.: Analysis of ESR1 and PIK3CA mutations in plasma cell-free DNA from ER-positive breast cancer patients. Oncotarget 2017;8:52142-52155.

24 Wardell SE, Nelson ER, Chao CA, McDonnell DP: Bazedoxifene exhibits antiestrogenic activity in animal models of tamoxifen-resistant breast cancer: implications for treatment of advanced disease. Clin Cancer Res 2013;19:2420-2431.

25 Wardell SE, Ellis MJ, Alley HM, et al.: Efficacy of SERD/SERM hybrid-CDK4/6 inhibitor combinations in models of endocrine therapy-resistant breast cancer. Clin Cancer Res 2015;21:5121-5130.

26 Fanning SW, Dharmarajan V, Mayne CG, et al.: Bazedoxifene inhibits ESR1 somatic mutants with improved potency compared to tamoxifene and raloxifene. Cancer Res 2016;76(suppl):abstr 4854.
27 Garner F, Shomali M, Paquin D, et al.: RAD1901: a novel, orally bioavailable selective estrogen receptor degrader that demonstrates antitumor activity in breast cancer xenograft models. Anticancer Drugs 2015;26:948-956.

28 Bardia A, Kabos P, Elledge R, et al.: Evaluation of RAD1901, a novel investigational, selective estrogen receptor degrader (SERD), for the treatment of ERpositive (ER+) advanced breast cancer. J Clin Oncol 2017;35(suppl):abstr 1014

29 Lai A, Kahraman M, Govek S, et al.: Identification of GDC-0810 (ARN-810), an orally bioavailable selective estrogen receptor degrader (SERD) that demonstrates robust activity in tamoxifen-resistant breast cancer xenografts. J Med Chem 2015;58:4888-4904.

30 Joseph JD, Darimont B, Zhou W, et al.: The selective estrogen receptor downregulator GDC-0810 is efficacious in diverse models of ER+ breast cancer. Elife 2016;5:e15828.

31 Hui R, Cornish AL, McClelland RA, et al.: Cyclin D1 and estrogen receptor messenger RNA levels are positively correlated in primary breast cancer. Clin Cancer Res 1996;2:923-928.

32 Finn RS, Dering J, Conklin D, et al.: PD 0332991, selective cyclin D kinase 4/6 inhibitor, preferentially inhibits proliferation of luminal estrogen receptorpositive human breast cancer cell lines in vitro. Breast Cancer Res 2009;11:R77. 